\title{
A structural analysis method for plastics (SAMP) based on injection molding and microstructures
}

\author{
Bingyun Jiang ${ }^{1,2, a}$, Li Li ${ }^{2}$ and Huilin Huang ${ }^{1}$ \\ ${ }^{1}$ School of Mechanical Engineering, Shanghai Jiao Tong University, Shanghai 200240, China \\ ${ }^{2}$ Co-Sim Team, Shanghai Prinfo Information Technology Co., Ltd., Shanghai 201203, China
}

\begin{abstract}
An advanced Structural Analysis Method for fiber filled Plastics (SAMP) based on injection molding and their microstructures was proposed. This proposed SAMP combines injection molding and microstructures together to analyze the mechanical behavior considering the fiber orientation and anisotropic materials. The fiber orientations are obtained through injection molding simulation, and the nonlinear anisotropic materials are obtained through mean-field homogenization at the microstructures. This SAMP are classed into 3 modules: material analysis, injection analysis and structure analysis. Both of the material analysis and injection analysis are coupled together into subsequent structure analysis to obtain reasonable and more accurate simulation results.
\end{abstract}

Keywords: structural analysis; plastics; fiber orientation; injection molding; microstructure.

\section{Introduction}

Many connector are used in almost all electronic products to connect signal or transmit current, such as MQS, CE-Box, Sim-Card and HDMI. They generally consist of housing and terminals, as shown in Fig. 1. Because the thermoplastics reinforced with short glass fiber could provide good mechanical strength to ensure they are safe enough, the housing are made of plastics, and are also increasingly used in many industrial applications.

Currently, depending on the market driven trends with many complex designs and shorter development cycles, the finite element method (FEM) is extensively used as a powerful tool in simulation and optimization of many engineering problems[1-2].Current FEM engineers neglect the effects of fiber orientation during injection molding processing. However, this fiber orientation determine material performance of housing. The finite element analysis of injection molded structures made of nonlinear anisotropic behavior of reinforced polymer is increasingly complex. In this case, the material behavior can significantly vary from one part to another of the structure and even from one integration point to the next in the plane and across the thickness of the structure due to the fiber orientation induced by the polymer flow [3].

\footnotetext{
a Corresponding author : Bingyunjiang@sjtu.edu.cn
} 


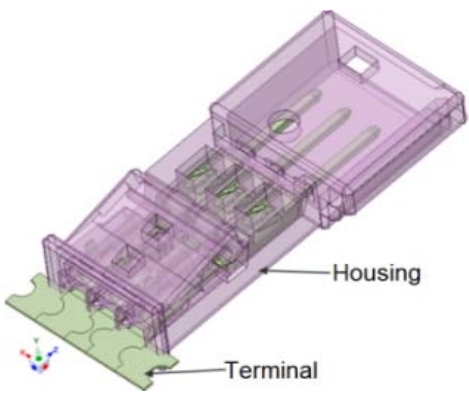

(a) MQS connector

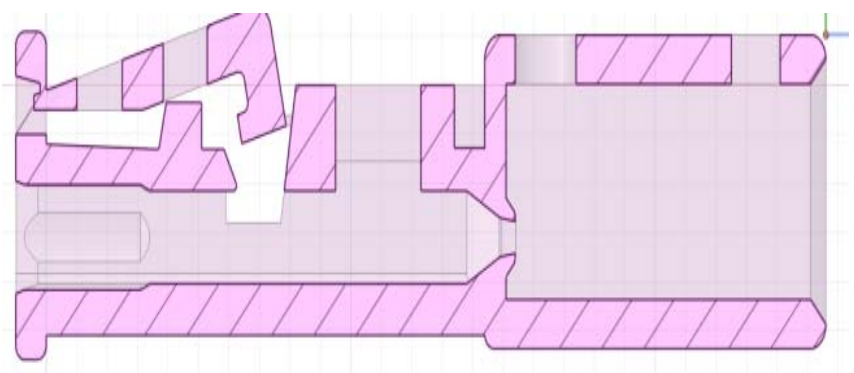

(b) MQS Housing

Figure 1. MQS connector and its housing

Knowledge of the material behavior is an essential ingredient of a FEM. So the conventional simulation results are unreasonable and inaccurate in the present time. In order to analyse the mechanical behavior of fiber filled plastics of connector reasonably and accurately, with considering fiber orientation and anisotropy material, an advanced Structure Analysis Method for fiber filled Plastics (SAMP) is proposed. This micro-macro scale transition approach aims to link the short fiber composite's macro response and its microstructure, and be classed into 3 modules: material analysis, injection analysis and structure analysis.

This SAMP based on injection molding and microstructures is established to analyse the structural strength of fiber filled plastics. Its technical or business value can be used: 1) Injection molding for the parts with complex shapes inside of which short fibers are dispersed. This SAMP could establish the anisotropy material model based on fiber orientation due to injection; 2) Analysing the micro-scale deformation of fiber and matrix; 3) Analysing macro-scale mechanical property of plastics reasonably and accurately, etc.

\section{SAMP methods and results}

\subsection{First module: material analysis}

In the material analysis module, the mean-field homogenization approaches based on Mori-Tanaka model is used to compute the mechanical properties of a composite as a function of its microstructure morphology, i.e., fiber diameter and length, orientation, mass fraction and micro matrix and fiber material behavior [4].

The major difficulty in the two-scale (and more generally multi-scale) approach is to solve this representative volume element (RVE) problems. In this section we give some very useful general averaging results. The notation and results are given hereafter.

Classical continuum mechanics analysis is carried out at macro level. At each macro point X, we know the macro strain $\mathrm{E}(\mathrm{X})$ and need to compute the macro stress or vice-versa.

The average quantity over a RVE (domain, volume V) is defined as described in equation (1):

$$
F(X, x)=\frac{1}{V} \int_{w} f(X, x) d V
$$

Where integration is performed with respect to micro coordinates, and $f(X, x)$ is the micro field in theRVE.

\subsection{Second module: injection analysis}

In the injection analysis module, the fiber orientation is obtained by injection molding simulation [5]. For example, the fiber distribution of bracket about 1 or 2 gates injection are shown in Fig.2. 


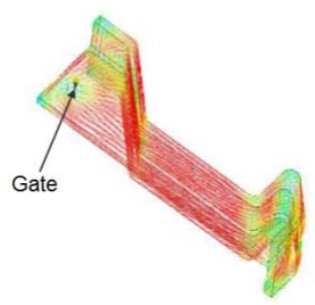

(a) Single gate injection

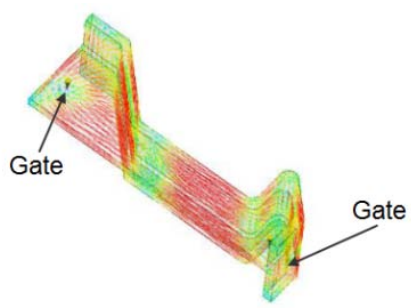

(b) Double gates injection

Figure 2. Fiber orientation distribution after injection molding simulation

Then we translate the fiber orientation from injection molding into an optimized structure mesh through mapping the data at integration points as Fig.3.

\subsection{Third module: structure analysis}

In the structure analysis module, the anisotropy material and mapped fiber orientation are coupled into structure simulation during iteration solving. For example, the bracket strength analysis molding is shown in Fig.4 (a), and its simulation results based on different material or fiber orientation are shown in Fig. 4(b).

In Fig. 4(b), the 1-gate and 2-gate curves are obtained by SAMP with considering fiber orientation and anisotropy material; the reaction force of 1-gate is larger than 2-gate due to glass fiber uniformity at the centre of bracket. Moreover, the 0-dir., 45-dir. and 90-dir. curves are listed by conventional approach based on 0-dir., 45-dir. and 90-dir. uniaxial stress-strain diagram. Both of 1-gate and 2-gate simulation curves are reasonable between the 0-dir. and 45-dir curves, because of their fiber distribution are almost in the range of $0 \sim 45$ direction.

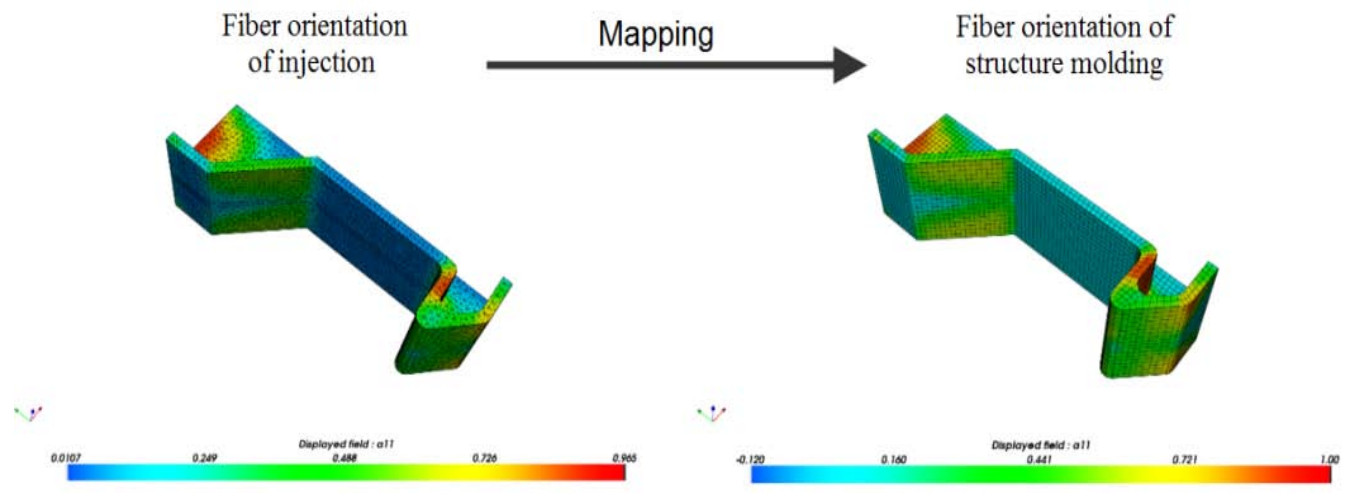

Figure 3. Mapping fiber orientation from injection model to structure model 


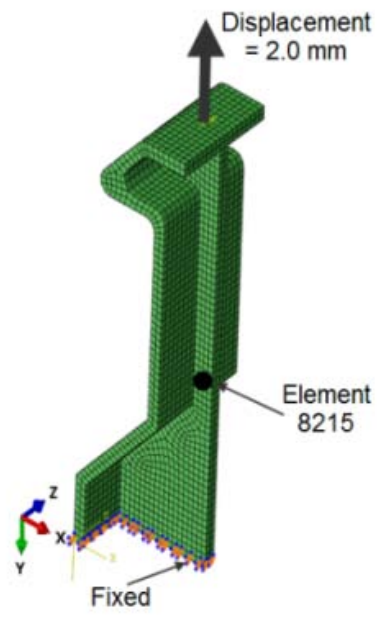

(a) Structure analysis modeling

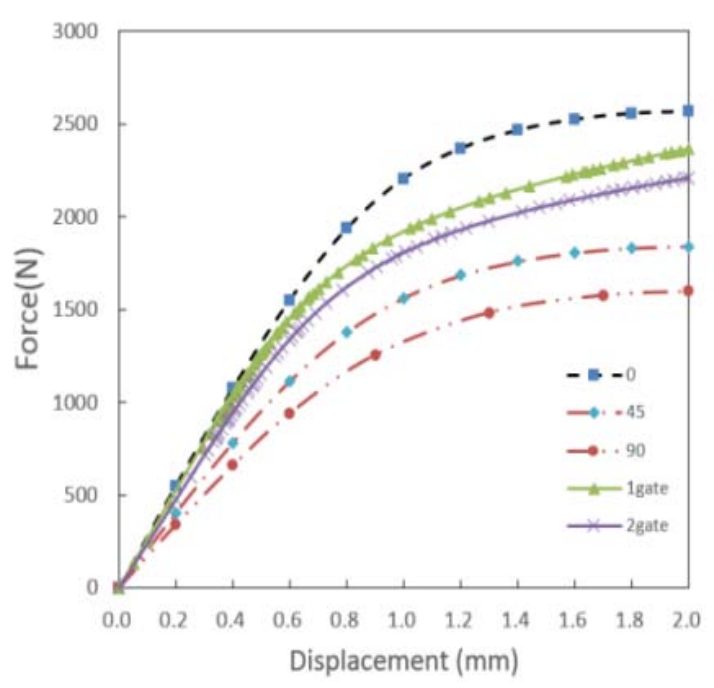

(b) Structure simulation results

Figure 4. Structure analysis results

\subsection{Microstructures}

At the same time, the microstructures behavior of fiber materials can be evaluated accurately using FEM of realistic Representative Volume Element (RVE) [6]. In the first step, the effective properties of the composite were obtained by FEA of an RVE of the microstructure. In the second step, the nonlinear composite behavior was analysed by linearization of the local behavior through the use of the tangent or secant stiffness tensors of the phases. The overall composite stiffness tensor was computed from those of each phase by the Mori-Tanaka approximation [7-8].

For example, the stress and strain distribution of fiber at the 8215 element point of bracket from Fig.4 (a) are analysed. The deformation model composite made up of a random and homogeneous dispersion of aligned, elastic ellipsoids embedded in an elastic-plastic matrix was simulated by means of computational micromechanics and homogenization methods. From the Fig. 5, we know that the deformation and stress have great different due to fiber orientation from different injection molding. Because the fiber of Fig. 5(a) and force have almost same direction, and the Fig. 5(b) and force have almost vertical direction, the strength of Fig. 5(a) is bigger than the Fig. 5(b).
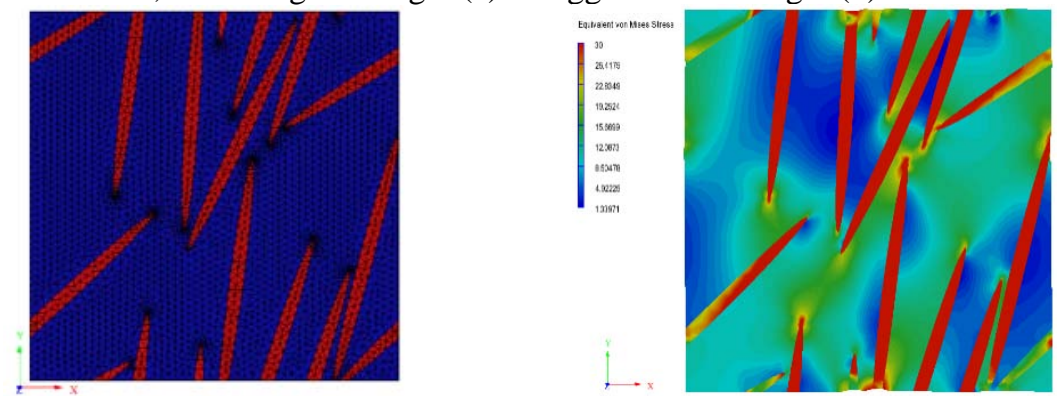

(a) 1-gate mesh and its stress distribution 

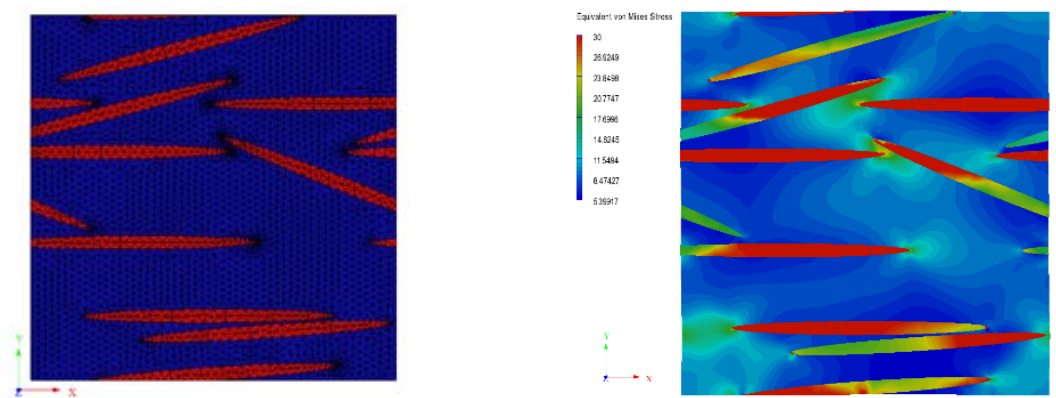

(b) 2-gate mesh and its stress distribution

Figure 5. Stress distribution for fiber reinforced materials at element of 8215

\section{Discussion and conclusion}

\subsection{Discussion}

Today, many parts in connector are made of plastics materials. The plastics parts are manufactured with injection molding technology. The mechanical properties is dependent by material intensely, and plastics material behavior is:1) dependent on the process settings during injection molding highly;2) anisotropic and inhomogeneous.

The conventional simulation results are unreasonable and inaccurate in the present time, so an advanced Structure Analysis Method for fiber filled Plastics (SAMP) is proposed.

\subsection{Conclusion}

This SAMP method has been used successfully to simulate the elastoplastic behavior of short-glass fiber reinforced plastics. Numerical predictions were compared with different injection proposals and different fiber orientations.

This SAMP based on injection molding and microstructures is established to analyse the fiber filled plastics, and its technical or business value can be used to: 1) establish the anisotropy material model based on fiber orientation;2) analyse the micro deformation of fiber based on RVE; 3) analyse macro mechanical property of plastics reasonably and accurately, etc. Of course, the damage in fiber reinforced thermoplastic materials typically produced by injection molding should be evaluated in the future.

\section{Acknowledgements}

The authors acknowledge the support of the National Natural Science Foundation of China (Grant Nos.51175335 and 51305261), and thank the great technical assistance from co-sim team of Shanghai Prinfo Information Technology Co., Ltd.

\section{References}

1. Hsu Y L, Hsu Y C, and Hsu M. S. Shape Optimal Design of Contact Springs of ElectronicConnectors [J].Journal of Electronic Packaging, 2002, 124(3):178-183.

2. Weight B L., Mattson C A, Magleby S P, et al. Configuration Selection, Modeling, and Preliminary Testing in Support of Constant Force Electrical Connectors [J]. Journal of ElectronicPackaging, 2007,129 (3): 236-246.

3. Hartl A M, Jerabek M, Lang R W. Effect of fiber orientation, stress state and notch radius on the 
impact properties of short glass fiber reinforced polypropylene[J]. Polymer Testing, 2015, 43: 1-9.

4. Wu L, Sket F, Molina-Aldareguia J M, et al. A study of composite laminates failure using an anisotropic gradient-enhanced damage mean-field homogenization model [J]. Composite Structures, 2015, 126: 246-264.

5. Peng X, Qin J, Jiang Y. An Approach for Predicting Fiber Orientation Distribution in Plastic Injection Molding of Composites [J]. British Journal of Applied Science \& Technology, 2015, 7(2): 186.

6. Tian W, Qi L, Zhou J, et al. Representative volume element for composites reinforced by spatially randomly distributed discontinuous fibers and its applications [J]. Composite Structures, 2015.

7. O. Pierard, C. Gonza'lez, J. Segurado, et al. Micromechanics of elasto-plastic materials reinforced with ellipsoidal inclusions [J]. International Journal of Solids and Structures,2007, 44: 6945-6962.

8. S. Kammoun, I. Doghri, L. Brassart, L. Delannay. Micromechanical modeling of the progressive failure in short glass-fiberreinforced thermoplastics - First Pseudo-Grain Damage model [J]. Composites: Part A, 2015, 73:166-175. 